Supplement of Eur. J. Mineral., 32, 101-119, 2020

https://doi.org/10.5194/ejm-32-101-2020-supplement

(C) Author(s) 2020. This work is distributed under

the Creative Commons Attribution 4.0 License.

(c) (1)
European Journal of

Mineralogy

Open Access

Supplement of

\title{
Unusual silicate mineralization in fumarolic sublimates of the Tolbachik volcano, Kamchatka, Russia - Part 1: Neso-, cyclo-, ino- and phyllosilicates
}

Nadezhda V. Shchipalkina et al.

Correspondence to: Nadezhda V. Shchipalkina (estel58@yandex.ru)

The copyright of individual parts of the supplement might differ from the CC BY 4.0 License. 


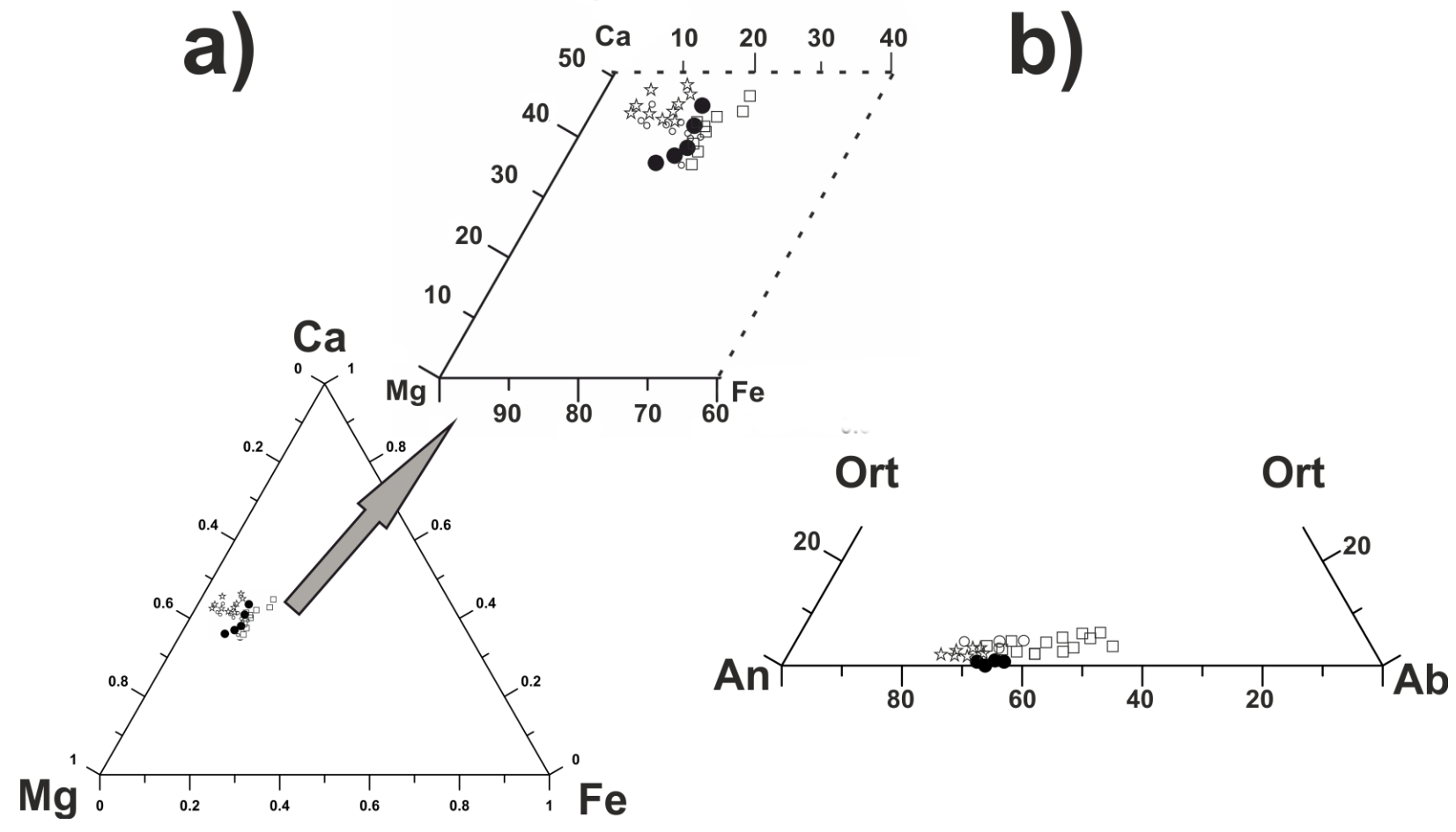

Fig. S1. Chemical features for Ca-Mg-Fe pyroxenes (a, with magnified fragment) and feldspars (b) from basalts of Great Fissure Tolbachik Eruption 1975-1976: • - our data; - data for magnesium basalts from (Fedotov and Markhinin, 1983); $\square-$ data for subalkaline alumina-rich basalts from (Fedotov and Markhinin, 1983). 
Table S1. Chemical composition of minerals and glass composing non-altered by fumarolic gas basalt scoria which hosts the Arsentnaya fumarole

\begin{tabular}{|c|c|c|c|c|c|c|c|c|c|c|c|}
\hline Component & Ol & Cpx & Cpx & Cpx & Cpx & Cpx & PI & PI & PI & PI & Glass \\
\hline $\mathrm{SiO}_{2}$ & 41.86 & 50.39 & 45.87 & 50.45 & 47.45 & 48.63 & 50.98 & 50.15 & 50.30 & 50.85 & 53.09 \\
\hline $\mathrm{TiO}_{2}$ & & 1.05 & 1.82 & 0.71 & 1.40 & 1.34 & & & & & 1.45 \\
\hline $\mathrm{Al}_{2} \mathrm{O}_{3}$ & & 3.66 & 8.03 & 3.12 & 5.63 & 4.27 & 31.61 & 32.00 & 31.96 & 31.19 & 15.17 \\
\hline $\mathrm{Cr}_{2} \mathrm{O}_{3}$ & & 0.13 & & 0.43 & & & & & & & \\
\hline $\mathrm{FeO} * 1$ & 0.77 & 9.33 & 8.87 & 8.24 & 9.28 & 9.94 & 0.99 & 1.05 & 0.99 & 1.08 & 12.99 \\
\hline $\mathrm{MnO}$ & 0.35 & 0.32 & 0.20 & 0.21 & 0.23 & 0.26 & & & & & \\
\hline $\mathrm{NiO}$ & 0.24 & & & & & & & & & & \\
\hline $\mathrm{CuO}$ & 0.38 & & & & & & & & & & \\
\hline $\mathrm{MgO}$ & 56.23 & 16.18 & 12.87 & 17.63 & 13.95 & 15.14 & 0.24 & 0.23 & 0.26 & 0.23 & 4.26 \\
\hline $\mathrm{CaO}$ & 0.16 & 17.42 & 20.47 & 17.17 & 19.17 & 17.80 & 13.19 & 13.71 & 13.53 & 12.80 & 5.15 \\
\hline $\mathrm{Na}_{2} \mathrm{O}$ & & 0.36 & 0.30 & 0.19 & 0.33 & 0.38 & 3.73 & 3.39 & 3.58 & 3.87 & 5.02 \\
\hline $\mathrm{K}_{2} \mathrm{O}$ & & 0.07 & & & & & 0.17 & 0.13 & & 0.13 & 1.23 \\
\hline $\mathrm{P}_{2} \mathrm{O}_{5}$ & & & & & & & & & & & 0.38 \\
\hline Total & 99.99 & 98.91 & 98.43 & 98.15 & 97.41 & 97.76 & 100.91 & 100.66 & 100.62 & 100.15 & 98.74 \\
\hline & \multicolumn{10}{|c|}{ Empirical formulae } & \\
\hline $\mathrm{Si}$ & 0.99 & 1.89 & 1.75 & 1.89 & 1.82 & 1.85 & 2.31 & 2.28 & 2.28 & 2.32 & \\
\hline $\mathrm{Ti}$ & & 0.03 & 0.05 & 0.02 & 0.04 & 0.04 & & & & & \\
\hline $\mathrm{Al}$ & & 0.16 & 0.36 & 0.14 & 0.25 & 0.19 & 1.68 & 1.71 & 1.71 & 1.67 & \\
\hline $\mathrm{Cr}$ & & 0.00 & & 0.01 & & & & & & & \\
\hline $\mathrm{Fe}^{2+}$ & 0.02 & 0.29 & 0.28 & 0.26 & 0.30 & 0.32 & 0.04 & 0.04 & 0.04 & 0.04 & \\
\hline $\mathrm{Mn}$ & 0.01 & 0.01 & 0.01 & 0.01 & 0.01 & 0.01 & & & & & \\
\hline $\mathrm{Ni}$ & 0.00 & & & & & & & & & & \\
\hline $\mathrm{Cu}$ & 0.01 & & & & & & & & & & \\
\hline $\mathrm{Mg}$ & 1.98 & 0.90 & 0.73 & 0.99 & 0.80 & 0.86 & 0.02 & 0.02 & 0.02 & 0.02 & \\
\hline $\mathrm{Ca}$ & 0.00 & 0.70 & 0.83 & 0.69 & 0.79 & 0.73 & 0.64 & 0.67 & 0.66 & 0.62 & \\
\hline $\mathrm{Na}$ & & 0.03 & 0.02 & 0.01 & 0.03 & 0.03 & 0.33 & 0.30 & 0.31 & 0.34 & \\
\hline $\mathrm{K}$ & & 0.00 & & & & & 0.01 & 0.01 & & 0.01 & \\
\hline$\Sigma_{c a t}$ & 3.01 & 4.02 & 4.03 & 4.02 & 4.03 & 4.03 & 5.02 & 5.02 & 5.02 & 5.02 & \\
\hline $\mathrm{O}(\mathrm{BoFC})$ & 4 & 6 & 6 & 6 & 6 & 6 & 8 & 8 & 8 & 8 & \\
\hline
\end{tabular}

$\mathrm{Ol}$ - olivine (forsterite), $\mathrm{Cpx}$ - clinopyroxene (Al- and Fe-bearing diopside), $\mathrm{Pl}$ - plagioclase (labrador); empty cell means content below detection limit; $\Sigma_{c a t}=$ sum of metal cations $+\mathrm{Si} ; \mathrm{O}(\mathrm{BoFC})$ is a basis of formula calculation, number of $\mathrm{O}$ atoms per formula unit; *1all iron is calculated as $\mathrm{Fe}^{2+}$. 
Table S2. Typical chemical composition of indialite (Ind), enstatite (En), diopside (Di), fluorophlogopite (FPhlg) from Mountain 1004.

\begin{tabular}{|c|c|c|c|c|c|c|c|c|c|}
\hline Component & Ind & En & En & Di & FPhlg & FPhlg & FPhlg & FPhlg & FPhlg \\
\hline & \multicolumn{9}{|c|}{ wt. $\%$} \\
\hline $\mathrm{SiO}_{2}$ & 48.40 & 56.82 & 57.02 & 50.72 & 43.42 & 44.33 & 44.68 & 44.25 & 43.53 \\
\hline $\mathrm{TiO}_{2}$ & - & 0.08 & 0.14 & 0.48 & 2.12 & 1.18 & 1.19 & 0.69 & 1.75 \\
\hline $\mathrm{Al}_{2} \mathrm{O}_{3}$ & 37.51 & 1.90 & 2.85 & 3.31 & 11.83 & 10.88 & 9.77 & 9.48 & 10.13 \\
\hline $\mathrm{Fe}_{2} \mathrm{O}_{3}$ & 0.55 & 2.74 & 2.61 & 5.76 & 1.65 & 1.38 & 5.94 & 3.46 & 5.75 \\
\hline MnO & - & 0.79 & 0.58 & 0.48 & - & - & - & 0.03 & - \\
\hline $\mathrm{CuO}$ & - & 0.28 & 0.34 & - & - & 0.20 & 2.16 & 5.86 & 0.44 \\
\hline $\mathrm{ZnO}$ & - & - & - & - & - & - & 0.73 & 1.08 & - \\
\hline MgO & 13.77 & 36.45 & 35.97 & 16.48 & 26.66 & 26.98 & 21.46 & 21.12 & 22.62 \\
\hline $\mathrm{CaO}$ & 0.06 & 1.43 & 1.12 & 21.31 & - & 0.10 & 0.08 & 0.06 & 0.12 \\
\hline $\mathrm{Na}_{2} \mathrm{O}$ & - & - & 0.16 & 0.76 & 0.86 & 0.84 & 0.42 & 0.45 & 0.49 \\
\hline $\mathrm{K}_{2} \mathrm{O}$ & - & - & 0.16 & - & 10.24 & 10.01 & 10.06 & 9.77 & 9.78 \\
\hline $\mathbf{F}$ & - & - & - & - & 7.83 & 8.06 & 8.27 & 8.25 & 8.15 \\
\hline Cl & - & - & - & - & 0.05 & 0.10 & 0.09 & 0.08 & 0.11 \\
\hline $\mathrm{O}=(\mathrm{F}, \mathrm{Cl})_{2}$ & & & & & 3.31 & 3.42 & 3.50 & 3.49 & 3.46 \\
\hline \multirow[t]{2}{*}{ Total } & 100.29 & 100.49 & 100.95 & 99.30 & 101.35 & 100.64 & 101.35 & 101.09 & 99.41 \\
\hline & \multicolumn{9}{|c|}{ Empirical formulae } \\
\hline $\mathbf{S i}$ & 4.73 & 1.93 & 1.92 & 1.86 & 3.02 & 3.10 & 3.17 & 3.18 & 3.11 \\
\hline $\mathbf{T i}$ & - & 0.00 & 0.00 & 0.01 & 0.11 & 0.06 & 0.06 & 0.04 & 0.09 \\
\hline Al & 4.32 & 0.08 & 0.11 & 0.14 & 0.97 & 0.90 & 0.82 & 0.80 & 0.85 \\
\hline $\mathrm{Fe}^{3+}$ & 0.04 & 0.07 & 0.07 & 0.16 & 0.09 & 0.07 & 0.32 & 0.19 & 0.31 \\
\hline Mn & - & 0.02 & 0.02 & 0.01 & - & - & - & 0.00 & - \\
\hline $\mathrm{Cu}$ & - & 0.01 & 0.01 & - & - & 0.01 & 0.12 & 0.32 & 0.02 \\
\hline Zn & - & - & - & - & - & - & 0.04 & 0.06 & - \\
\hline Mg & 2.00 & 1.84 & 1.81 & 0.90 & 2.77 & 2.81 & 2.27 & 2.27 & 2.41 \\
\hline $\mathrm{Ca}$ & 0.01 & 0.05 & 0.04 & 0.84 & - & 0.01 & 0.01 & 0.00 & 0.01 \\
\hline $\mathbf{N a}$ & - & - & 0.01 & 0.06 & 0.12 & 0.12 & 0.06 & 0.07 & 0.07 \\
\hline $\mathbf{K}$ & - & - & 0.01 & - & 0.91 & 0.89 & 0.91 & 0.90 & 0.89 \\
\hline $\mathbf{F}^{-}$ & - & - & - & - & 1.73 & 1.78 & 1.85 & 1.88 & 1.84 \\
\hline $\mathrm{Cl}^{-}$ & - & - & - & - & 0.01 & 0.01 & 0.01 & 0.01 & 0.01 \\
\hline$\Sigma_{c a t}$ & 11.09 & 4.00 & 4.00 & 3.99 & 7.99 & 7.97 & 7.76 & 7.83 & 7.77 \\
\hline BoFC & $18 \mathrm{O}$ & 60 & 60 & 60 & $* 1$ & $* 1$ & $* 1$ & $* 1$ & $* 1$ \\
\hline
\end{tabular}

Note. Dash means the content below the detection limit. $\Sigma_{c a t}=$ sum of metal cations $+\mathrm{Si}$. BoFC is a basis of formula calculation: number of $\mathrm{O}$ atoms per formula unit $(a p f u)$, except of micas $\left(*^{1}\right)$ for which $\mathrm{BoFC}$ is $\mathrm{O}+\mathrm{F}=$ 12 apfu. 
